\title{
Estrategia para fomentar la transferencia de conocimiento en una entidad del sector defensa*
}

[Artículos de investigación]

\author{
Diana Catherine Lugo Cobos** \\ Linda Marcela Lugo Mondragón ${ }^{* * *}$ \\ Diana Magally Suárez Yate***** \\ Guillermo Peña Guarín ${ }^{* * * * * *}$
}

\begin{abstract}
*Artículo de resultado de investigación
** Jefatura de Formación, Instrucción y Educación Naval de la Armada Nacional. Magíster en Calidad y Gestión Integral. Profesional en Ciencia de la Información y la Documentación, Bibliotecología y Archivística. Especialista en Gerencia de Calidad. Línea de investigación: Calidad y Gestión. Universidad Santo Tomás, Bogotá, Colombia. Correo electrónico: dianalugocobos@gmail.com; ORCID https://orcid.org/0000-0001-7034-6160; CVLAC: https://scienti.minciencias.gov.co/cvlac/visualizador/generarCurriculoCv.do?cod rh $=0$ 001813833

*** Universidad Central. Magíster en Calidad y Gestión Integral. Profesional en Administración de Empresas. Especialista en Gerencia de Calidad. Línea de investigación: Calidad y Gestión. Universidad Central, Bogotá, Colombia. Correo electrónico: lindalugo@gmail.com; ORCID https://orcid.org/0000-0001-9986-1115; CVLAC: https://scienti.minciencias.gov.co/cvlac/visualizador/generarCurriculoCv.do?cod_rh $=0$ 001814020

**** Universidad Santo Tomás. Magíster en Calidad y Gestión Integral. Contadora Pública. Especialista en Administración y Gerencia de Sistemas de la Calidad. Línea de investigación: Calidad y Gestión. Universidad Santo Tomás, Bogotá, Colombia. Correo electrónico: yatemagally@hotmail.com; ORCID https://orcid.org/0000-0002-42769550; CVLAC:

https://scienti.minciencias.gov.co/cvlac/visualizador/generarCurriculoCv.do?cod_rh $=0$ 001813898

${ }_{* * * * *}$ Universidad Santo Tomás. Magíster en Calidad y Gestión Integral. Ingeniero químico. Línea de investigación: Gestión del Conocimiento y Gestión Integral. Universidad Santo Tomás, Bogotá, Colombia. Correo electrónico: guillerpegu@yahoo.com; ORCID: https://orcid.org/0000-0003-3822-4010. CvLAC: http://scienti.colciencias.gov.co:8081/cvlac/visualizador/generarCurriculoCv.do?cod_r $\underline{\mathrm{h}=0000382361}$
\end{abstract}


Recibido: 17 de abril de 2020

Revisado: o1 de agosto de 2020

Aceptado: 9 de agosto de 2020

Cómo citar este artículo:

Lugo Cobos, D. C., Lugo Mondragón, L. M., Suárez Yate, D. M. y Peña Guarín, G. (2020). Estrategia para fomentar la transferencia de conocimiento en una entidad del sector defensa. Signos - Investigación en sistemas de gestión, 13(1). https://doi.org/10.15332/24631140.6344

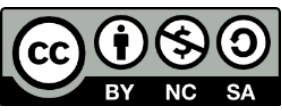

\section{Resumen}

El conocimiento y la experiencia de las personas impulsan el crecimiento en las organizaciones. Sin embargo, tras alcanzar un nivel de experticia, las personas pueden cambiar de organización o ser trasladadas a otro cargo, sin que el conocimiento se transfiera adecuadamente entre funcionarios, lo cual genera traumatismo en la gestión administrativa. Este artículo presenta una estrategia para transferir el conocimiento asociado a los procesos de las entidades públicas de una manera que permita preservarlo. El estudio se realizó con un enfoque mixto que incluyó una revisión de bibliografía sobre transferencia del conocimiento en español e inglés entre los años 2000 y 2018. Se aplicó un instrumento para diagnosticar la gestión del conocimiento con base en la teoría de Nonaka y Takeuchi. El informe final de las cuatro fases y los cinco facilitadores arrojó resultados por debajo de los esperados, lo que demostró que la entidad presenta falencias en esta área. Se elaboró una propuesta para fomentar la transferencia de conocimiento en la Jefatura y se validó con expertos. La fiabilidad del juicio se corroboró mediante el coeficiente de correlación alfa de Cronbach y el coeficiente de concordancia W de Kendall.

Palabras clave: gestión del conocimiento, transferencia del conocimiento, aprendizaje organizacional. 


\section{Strategy to promote knowledge transfer in a defense sector entity}

\section{Abstract}

People's knowledge and experience drive organizations growth. However, after reaching a level of expertise, people may change organizations or be transferred to another position, without knowledge being adequately transferred between staff members, which causes troubles in administrative management. This article presents a strategy for transferring the public entities processes related knowledge in a way that preserves it. It was a mixed approach study where a review of the literature on knowledge transfer in Spanish and English between 2000 and 2018 was included. A tool was applied to diagnose knowledge management based on Nonaka and Takeuchi theory. The final report of the four phases and the five facilitators yielded results below those expected, which demonstrated the entity shortcomings in that area. A proposal to promote knowledge transfer in the Management was developed and validated by experts. The reliability of the judgement was corroborated by Cronbach's alpha correlation coefficient and W Kendall's coefficient of concordance.

Keywords: knowledge management, knowledge transfer, organizational learning.

\section{Estratégia para promover a transferência de conhecimento em uma entidade do setor de defesa}

\section{Resumo}

O conhecimento e a experiência das pessoas impulsionam o crescimento nas organizações. Entretanto, após atingir um nível de experiência, as pessoas podem mudar de organização ou ser transferidas para outro cargo, sem que o conhecimento seja transferido adequadamente entre 
seus funcionários, levando a traumas na gestão administrativa. Este artigo apresenta uma estratégia de transferência do conhecimento associado aos processos das entidades públicas de uma forma que permita sua preservação. $O$ estudo foi conduzido com uma abordagem mista incluindo uma revisão da bibliografia especializada sobre transferência de conhecimentos em espanhol e inglês entre 2000 e 2018. Uma ferramenta foi aplicada para diagnosticar a gestão do conhecimento baseado na teoria de Nonaka e Takeuchi. O relatório final das quatro fases e os cinco facilitadores mostraram resultados abaixo das expectativas, demonstrando as deficiências da entidade nesta área. Uma proposta para promover a transferência de conhecimentos na chefia foi desenvolvida e validada com especialistas. A confiabilidade da análise foi corroborada pelo coeficiente de correlação alfa de Cronbach e pelo coeficiente de concordância W de Kendall.

Palavras-chave: gestão do conhecimento, transferência de conhecimento, aprendizagem organizacional.

\section{Introducción}

El conocimiento es el modo en que las personas y las entidades dan sentido a la experiencia; es un factor significativo que proporciona valor a la organización al permitir que las operaciones se desarrollen dentro de los parámetros definidos y generar a futuro nuevas oportunidades de crecimiento. Sin embargo, el conocimiento, la información, el entendimiento, las competencias, los valores y las actitudes, por lo general, no figuran dentro de los estados financieros. Según Vázquez (2017), la transferencia es el intercambio del conocimiento en una forma de tecnología, método o herramienta, a través de un producto o servicio, mientras la aplicación es la utilidad de la transferencia, que da lugar a una innovación.

Romero (2018) afirma que la gestión del conocimiento es un concepto que se refiere a la acción que muchas empresas realizan para transferir el 
conocimiento desde el lugar en que se origina hasta el sitio en que va a utilizarse. Fomentar la transferencia de conocimiento implica desarrollar estrategias al interior de las organizaciones, así como identificar las competencias de cada integrante y aplicar en forma consistente la capacidad de hacer y la capacidad de aprender, para que dicho conocimiento se comparta y se utilice de manera adecuada entre los miembros de la misma organización (Engelbrecht et ál., 2019, Torres y Rojas, 2017). La competencia organizacional cada vez se presenta más entre los trabajadores de las organizaciones, debido a que estas los empoderan cada día desde diferentes teorías y estrategias para ser grandes líderes, gestores y competidores en el sector en el que desarrollan sus habilidades profesionales.

Por otro lado, la norma ISO 9001:2015 contiene los requerimientos que se pueden asimilar a los componentes de la gestión del conocimiento; no obstante, el sistema de gestión de la calidad por sí solo no garantiza una adecuada gestión del conocimiento organizacional que favorezca la producción de información útil para establecer el direccionamiento estratégico, que consolide buenas prácticas y que soporte la innovación de procesos, productos y servicios.

En su artículo sobre la transferencia del conocimiento en las empresas, Santamaría y Cárdenas (2016) señalan que el conocimiento es la base de los seres humanos porque permite mejorar la calidad de vida en todos sus ámbitos. Los autores detallan los principales factores que inciden en las empresas para que estas puedan transferir el conocimiento de una forma adecuada. Marulanda et ál. (2018) afirman que la transferencia del conocimiento en las organizaciones no recae directamente en los trabajadores, sino en las empresas, que deben implementar estrategias y generar cultura organizacional y políticas de gestión y transferencia de 
conocimiento que permitan realizar un buen intercambio de conocimiento y experiencias entre los equipos de trabajo que las integran.

Actualmente, un problema que surge en el sector defensa son las necesidades del servicio y las circunstancias de interés vinculadas a la eficacia y eficiencia de las instituciones del sector. Estas pueden suprimir cargos, efectuar nombramientos provisionales, realizar ascensos, desvincular o retirar a los funcionarios de sus cargos o rotar de cargo al personal militar y civil, de manera inesperada. Esto conduce a que las personas interrumpan su trabajo de manera abrupta y se marchen con el conocimiento y experiencia adquirida en el tiempo del ejercicio de su labor. Por consiguiente, para estas entidades es un gran reto asegurar la transferencia del conocimiento y mitigar su fuga, para que de esta manera no resulten afectadas la productividad y el cumplimiento de los objetivos institucionales.

Las principales vulnerabilidades en la transferencia de conocimiento se pueden identificar en la cultura organizacional, la gestión administrativa, los procesos de manejo y control de la documentación, la entrega de los cargos y el desarrollo de las actividades de inducción, que no logran evitar la fuga del conocimiento ni facilitan su preservación y acceso.

Por consiguiente, es necesario fomentar estrategias que coadyuven al intercambio de conocimiento para mejorar los saberes y la experiencia, faciliten su transferencia al momento de abandonar un cargo y entregarlo al sucesor, incentiven en los funcionarios su capacidad para ser difusores de los conocimientos adquiridos, y desarrollen hábitos para el intercambio del conocimiento.

El objetivo del presente artículo es presentar los resultados de la investigación en la que se planteó una estrategia para fomentar la transferencia del conocimiento en la Jefatura de Formación, Instrucción y 
Educación Naval de la Armada Nacional (Jinen), sede Bogotá, con el propósito de mitigar la pérdida de conocimiento e impedir que las operaciones administrativas se vean afectadas. Esta propuesta se podría hacer extensiva a otras áreas de la Armada Nacional.

\section{Metodología}

Para la investigación, se adoptó un enfoque mixto (cualitativocuantitativo), que incluyó una revisión de la literatura en diferentes fuentes bibliográficas, la elaboración de la propuesta de la estrategia, la aplicación del instrumento diagnóstico tipo encuesta y la validación de contenido de la estrategia propuesta para el proyecto. El alcance de la investigación fue de tipo descriptivo, teniendo en cuenta que se observó un fenómeno y se describieron sus variables. Se trabajó con un método inductivo, que se basó en un proceso de explorar, analizar, describir y generar perspectivas teóricas.

Se indagó en diferentes fuentes de información acerca de estrategias de transferencia del conocimiento en las organizaciones y se realizó una revisión sistemática exploratoria de literatura (figura 1). 
Figura 1. Revisión de literatura

Búsqueda de información

Bases de datos: ScienceDirect , Jstor, Scopus, Redalyc, Dialnet, SciELO

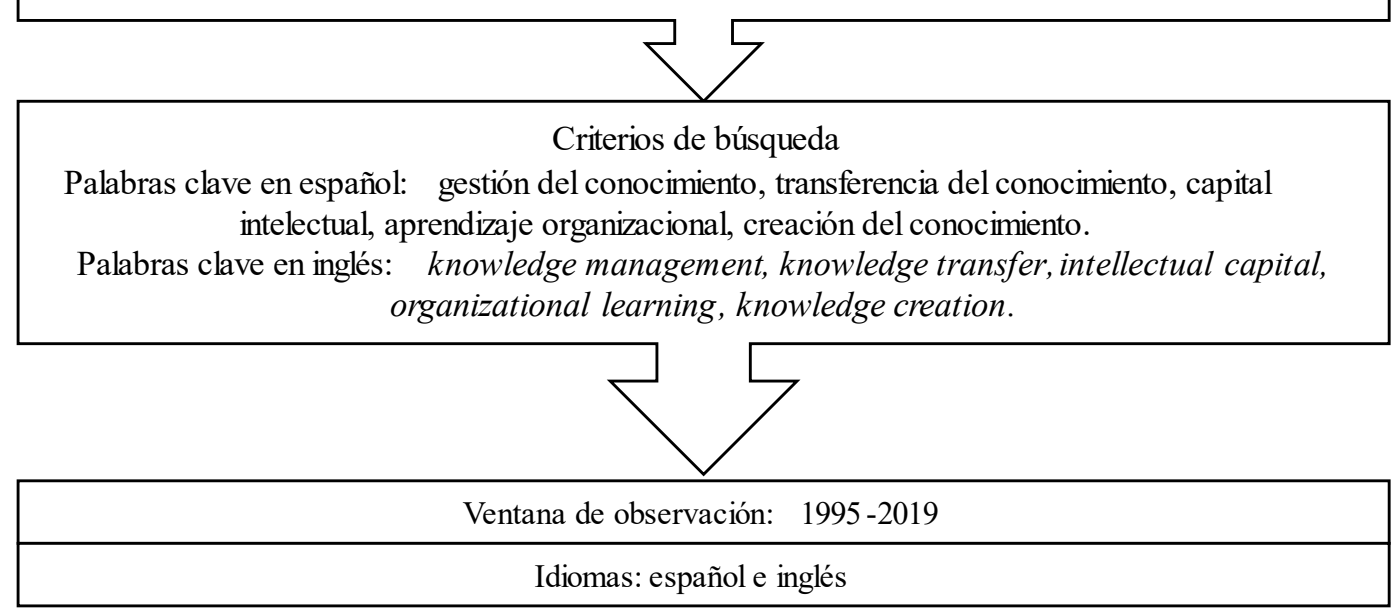

Fuente: elaboración propia.

Una vez identificados y revisados, los textos seleccionados se organizaron en una matriz documental por categorías (autor, título, publicado, año, resumen, fundamento teórico, concepto de transferencia del conocimiento, métodos de transferencia), con el objetivo de consolidarlos y validar su aporte a la elaboración del marco referencial y el planteamiento de la estrategia de transferencia de conocimiento.

Con el propósito de identificar los problemas y el estado en que se encontraba la transferencia del conocimiento al interior de la Jefatura, se llevó a cabo un diagnóstico mediante un instrumento construido a partir de la teoría de la creación del conocimiento organizacional (Peña, 2015). Se definió una muestra selectiva y por conveniencia de 20 funcionarios, a quienes se aplicó el instrumento (tabla 1). 
Tabla 1. Descripción de la muestra

\begin{tabular}{|l|l|c|}
\multicolumn{1}{|c|}{ Rango } & \multicolumn{1}{c|}{ Grados } & \multicolumn{1}{c|}{ Total } \\
\hline Oficiales & $\begin{array}{l}\text { Capitán de navío o corbeta y teniente de } \\
\text { navío } \\
\text { Suboficiales }\end{array}$ & Jefe técnico - Suboficiales jefes \\
\hline Infantes & Infantes de marina profesional & $\mathbf{5}$ \\
\hline Personal civil & $\begin{array}{l}\text { Asesora o profesional de defensa - Técnico } \\
\text { de servicios }\end{array}$ & $\mathbf{5}$ \\
\hline Total & & $\mathbf{5}$ \\
\hline
\end{tabular}

Fuente: elaboración propia.

El instrumento permitió identificar el comportamiento de las fases de la gestión del conocimiento (socialización, exteriorización, combinación e interiorización), el estado de los facilitadores organizacionales y el funcionamiento de los Ba o espacios de conocimiento, que se derivan de las categorías, variables e indicadores en los que está fundamentado el instrumento utilizado para el diagnóstico.

De acuerdo con la literatura analizada, la teoría seleccionada y los resultados generados del instrumento diagnóstico aplicado a los funcionarios de la Jefatura en relación con la gestión del conocimiento, a través de mesas de trabajo, se realizó la propuesta de la estrategia para fomentar la transferencia del conocimiento entre los servidores públicos con base en el diseño de una infografía. Se utilizó un diseño amigable y dinámico para establecer la estrategia según las cuatro fases de la teoría de Nonaka y Takeuchi (1995) y, así, facilitar la comprensión por parte del personal que integra la Jefatura.

Esta propuesta se sometió a una validación de contenido con el fin de determinar la correspondencia de los contenidos con respecto al dominio o campo que se deseaba medir (Urrutia, 2014), que en este caso fue la transferencia de conocimiento. La validación fue realizada por siete 
expertos que cumplían los criterios de educación, antigüedad en el sector y experiencia previamente definidos, y que aplicaron un instrumento validado en contextos similares (Rodríguez-Rojas, 2015). La fiabilidad del juicio de expertos se verificó posteriormente mediante la prueba de correlación del coeficiente alfa de Cronbach y el coeficiente de concordancia W de Kendall.

\section{Resultados y discusión}

Para recolectar información acerca de estrategias de transferencia del conocimiento en las organizaciones, se consultaron documentos publicados en las bases de datos ScienceDirect, Jstor, Scopus, Redalyc, Dialnet y SciELO entre 1995 y 2019, usando términos de búsqueda en español e inglés (figura 1). Teniendo en cuenta como criterio de inclusión que los artículos trataran sobre mejores prácticas para la trasferencia de conocimiento en las organizaciones (Manchado, 2009), se seleccionaron 200 artículos, de los cuales se revisaron 56 y se analizaron 20, que se emplearon como referentes para el estudio (figura 2). 
Figura 2. Identificación y selección de literatura de transferencia de conocimiento

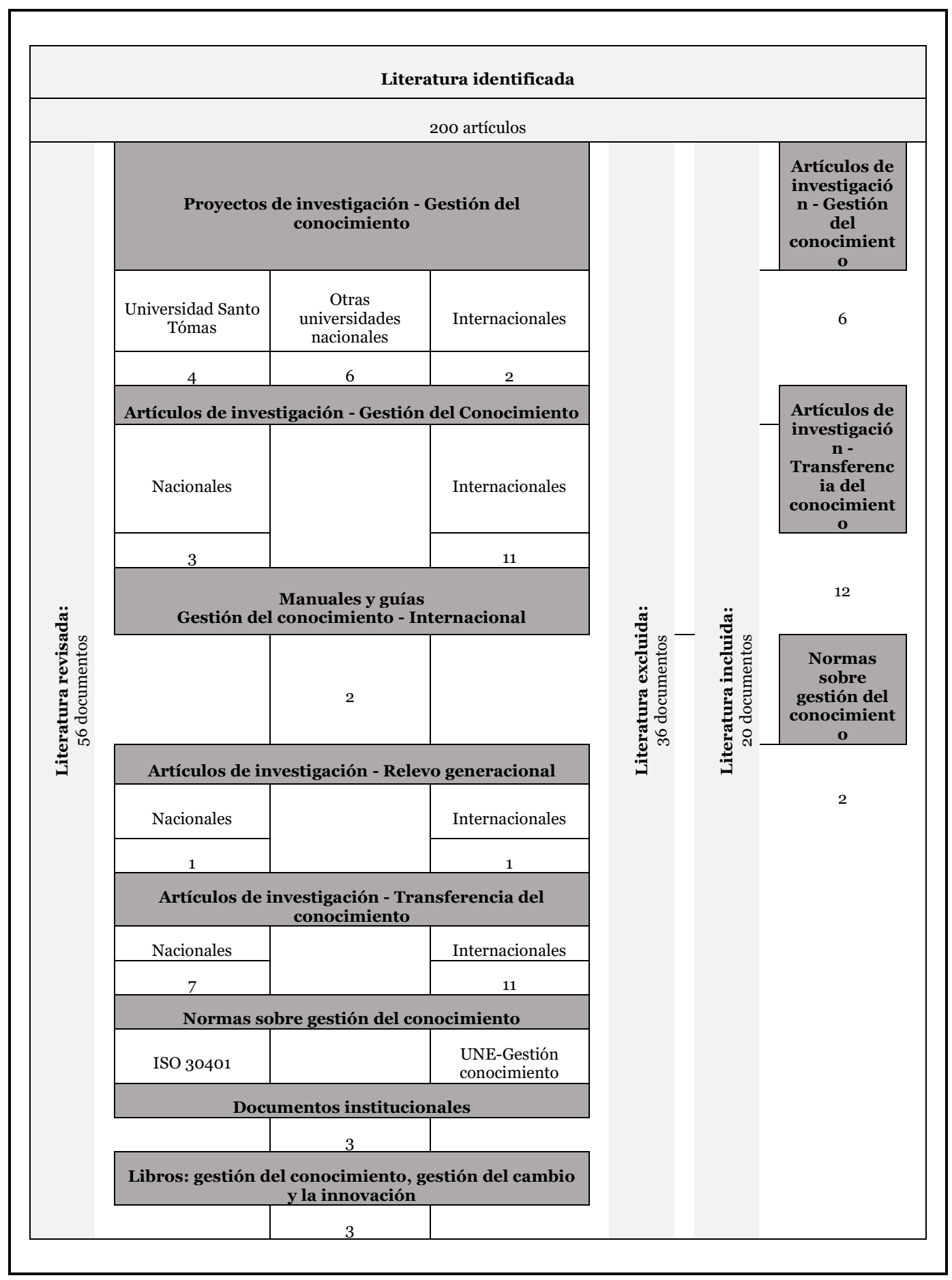

Fuente: elaboración propia.

SIGNOS, Investigación en Sistemas de Gestión

ISSN: 2145-1389 | e-ISSN: 2463-1140 | DOI: https://doi.org/10.15332/24631140

Vol. 13 N.o 1 | enero-junio de 2021 
De acuerdo con lo anterior, se seleccionó la teoría de la creación del conocimiento organizacional como referente principal para este trabajo (Nonaka y Takeuchi, 1995), debido a su enfoque sistémico, compatible con los sistemas de gestión basados en normas técnicas internacionales, y su orientación hacia la mejora continua (figura 3).

Figura 3. Modelo dinámico de la teoría de Nonaka y Takeuchi (1995)

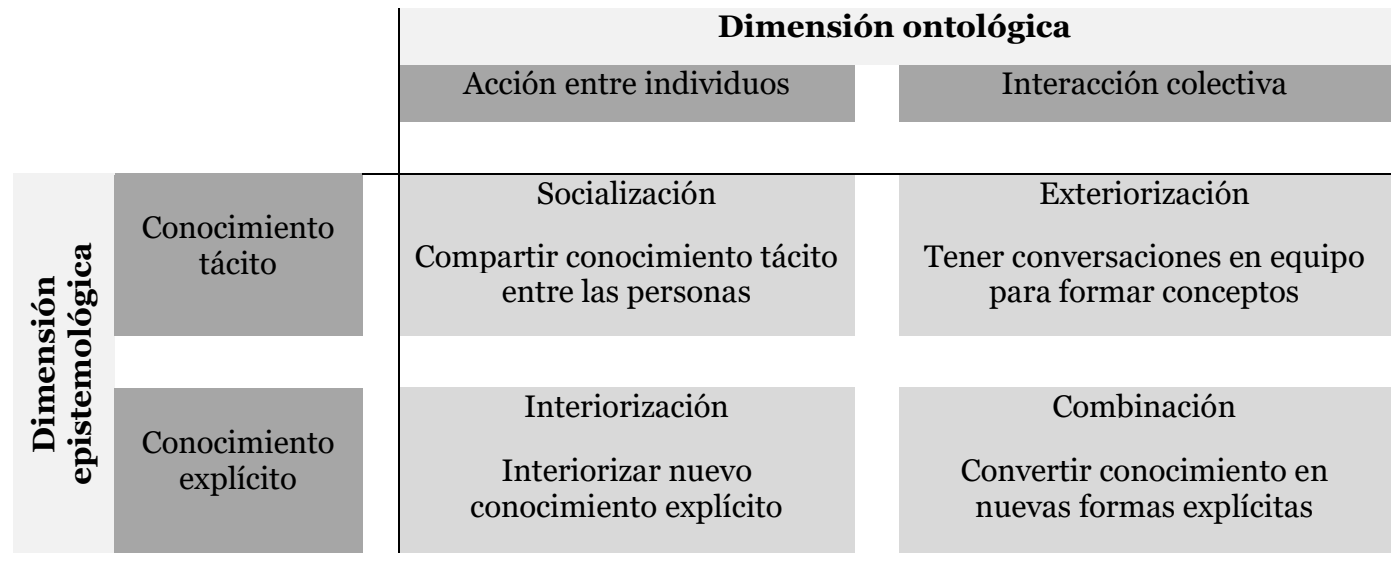

Fuente: adaptada de Bueno (2003).

Para identificar los problemas y el estado de transferencia del conocimiento al interior de la Jefatura, se aplicó un instrumento basado en la teoría de la creación del conocimiento (Peña, 2015) que contiene 42 preguntas con respuestas según la escala de Likert. Mediante este cuestionario se determinó el estado de las cuatro fases de creación del conocimiento, el nivel de desarrollo de los facilitadores organizacionales que propone dicha teoría como pilar para adelantar la gestión del conocimiento y la existencia de Ba o espacios de conocimiento en los que se lleva a cabo. La figura 4 muestra que la fase de socialización fue la mejor calificada y que las demás no alcanzaron a superar el umbral del $66 \%$, establecido como mínimo para identificar un desempeño satisfactorio. 
Figura 4. Resultados generales de las cuatro fases

-TOTALMENTE DE ACUERDO $\quad$ NIDE ACUERDO O DESACUERDO $\square$ TOTALMENTE EN DESACUERDO

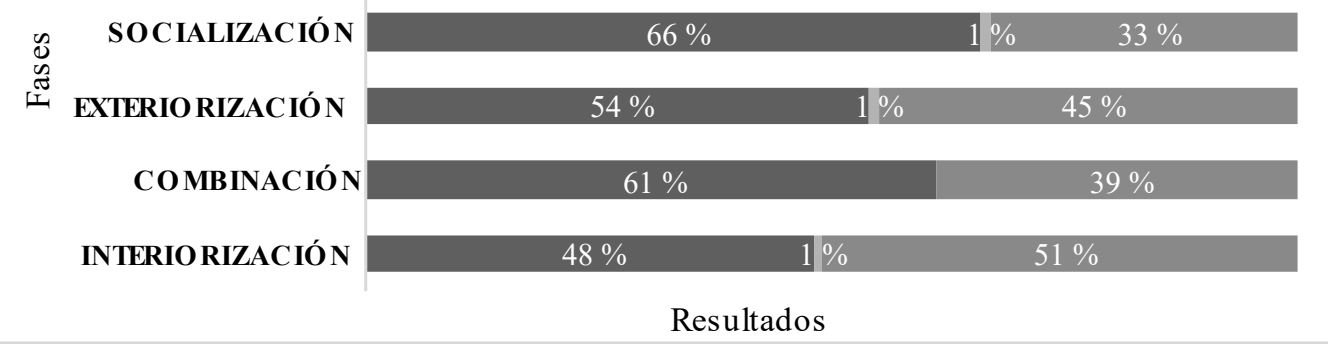

Fuente: elaboración propia.

De las cuatro fases, se observa que en la organización no se cumplen las de exteriorización, combinación e interiorización. Para el planteamiento de la estrategia de transferencia del conocimiento en la Jefatura de Formación, Instrucción y Educación Naval, se deberá incluir esas fases.

- $\quad$ Socialización: se destaca la disposición de los funcionarios para compartir sus conocimientos con el fin de mejorar el trabajo; sin embargo, la organización no facilita el tiempo ni los espacios para compartir el conocimiento.

- Exteriorización: se efectúa el análisis de los datos que se generan en los procesos con el fin de transformarlos en información útil; sin embargo, no hay participación activa de las personas ni se estimula el trabajo en equipo.

- Combinación: los medios de comunicación interna permiten mejorar el conocimiento de las personas. No se identifican mecanismos definidos para recopilar e incorporar las mejores prácticas de los procesos. 
- Interiorización: se encontró significativa la percepción de las personas sobre lecciones aprendidas cuando se han presentado fallas en los procesos; por otra parte, se cuestiona la evaluación de la eficacia de las capacitaciones que buscan asegurar que las personas tienen el conocimiento necesario para llevar a cabo sus funciones.

La figura 5 presenta los resultados que arrojó el diagnóstico en relación con los facilitadores organizacionales.

Figura 5. Resultados generales de los facilitadores

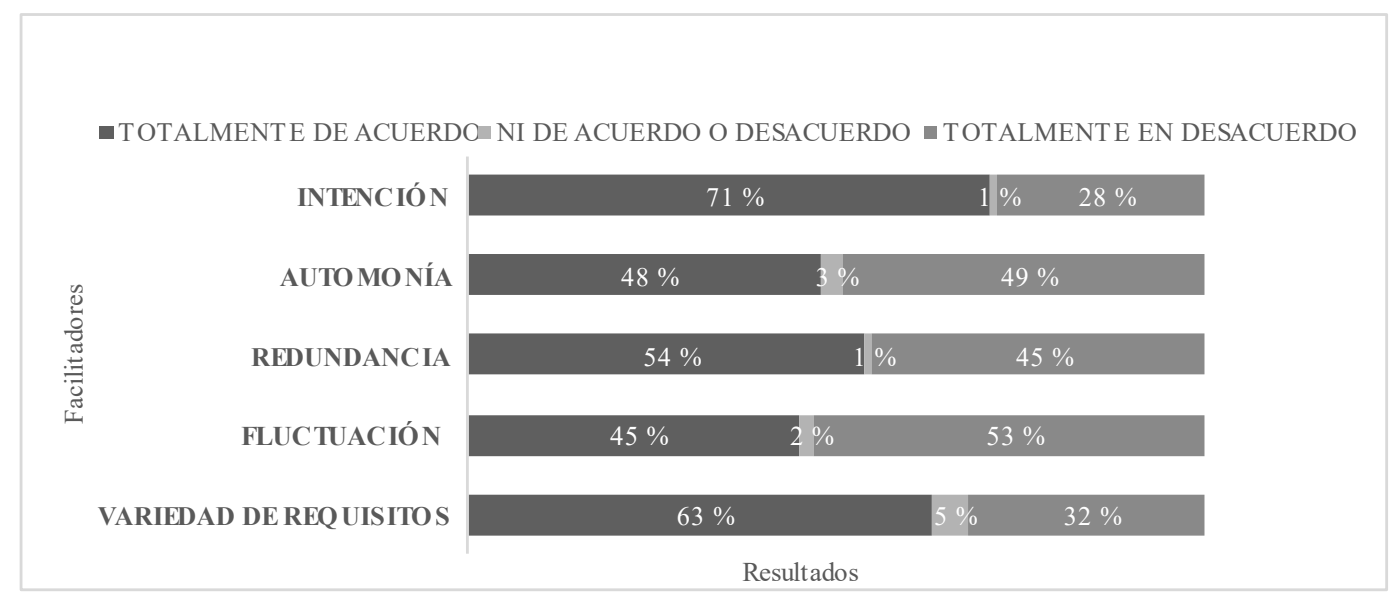

Fuente: elaboración propia.

Los facilitadores evalúan el contexto que suministra la organización para promover la gestión del conocimiento y las condiciones necesarias para que la espiral del conocimiento se accione. El facilitador de intención fue el mejor calificado, dada la claridad existente respecto a los objetivos que se persiguen con la gestión del conocimiento y cómo se articulan con la estrategia general. En los demás facilitadores, la Jefatura debe trabajar más.

Un aspecto interesante del diagnóstico fue la interpretación de los facilitadores al interior de esta entidad del sector defensa, ya que el facilitador de autonomía resulta inviable por la cultura y la estructura 
organizacional, dominada por jerarquías muy marcadas, por la antigüedad en el servicio y por el respeto a los superiores inmediatos. Además, se decidió que los facilitadores de fluctuación y caos creativo, redundancia y variedad de requisitos no se reflejaran directamente con estos nombres dentro de la propuesta, para hacerla comprensible y dinámica. Sin embargo, estos facilitadores estuvieron inmersos en las actividades de las diferentes fases.

Después, a fin de plantear una estrategia de transferencia de conocimiento para la Jefatura de Educación de acuerdo con las necesidades institucionales, se desarrollaron las fases del ciclo de la creación del conocimiento, a las cuales se les han modificado los nombres para hacerlas más afines a la cultura organizacional y facilitar su comprensión: difusión del conocimiento individual, interacción colectiva de conocimiento, combinación del conocimiento e interiorización de nuevo conocimiento. En cada fase se incluyeron los Ba o espacios de conocimiento como los lugares, físicos o virtuales, donde se desarrollan las fases de la gestión del conocimiento. Se decidió ilustrar la propuesta en forma de infografía, una práctica habitual en la organización, con un texto introductorio y explicativo. A continuación, se presenta la propuesta (tabla 2). 
Estrategia para fomentar la transferencia del conocimiento entre los servidores públicos de la Jefatura de Formación, Instrucción y Educación

Naval

El conocimiento es un activo intangible importante que poseen las personas, por medio del cual se genera valor a las entidades a través del mejoramiento de los procesos que realizan. Por ello, esta estrategia plantea fomentar la transferencia de conocimiento con el propósito de difundir y preservar dicho conocimiento asociado a los procesos, y así contribuir con buenas prácticas al aumento y fortalecimiento de las habilidades, competencias y capacidades de los funcionarios de la Jefatura de Formación, Instrucción y Educación Naval de la Armada Nacional.

Fuente: elaboración propia.

\section{Fase de socialización}

\section{Esta dimensión tiene como propósito identificar la acción entre} individuos, es decir compartir conocimiento tácito entre las personas (tabla 3).

Tabla 3. Fase de socialización

\begin{tabular}{ll}
\multicolumn{1}{c}{$\begin{array}{l}\text { Lograr que el conocimiento individual sea trasmitido y recibido entre los } \\
\text { servidores públicos para generar nuevos conjuntos de conocimientos y } \\
\text { experiencias. }\end{array}$} \\
$\begin{array}{ll}\text { Gestores de } \\
\text { transferencia del } \\
\text { conocimiento por } \\
\text { cada dirección }\end{array}$ & $\begin{array}{l}\text { Incluir en los manuales de funciones de los funcionarios que } \\
\text { conforman la Jefatura un compromiso concerniente a la apropiación } \\
\text { del conocimiento y su transferencia a todas las partes interesadas. }\end{array}$ \\
& $\begin{array}{l}\text { Incluir en el plan de capacitación programas de formación para el } \\
\text { personal que asumirá el rol de gestor de transferencia de } \\
\text { conocimiento. }\end{array}$ \\
& $\begin{array}{l}\text { Incluir en la orden del día el nombramiento como gestor de } \\
\text { transferencia de conocimiento de cada dirección a un funcionario que } \\
\text { será el enlace entre su equipo de trabajo y el director para recibir, } \\
\text { consolidar y promover acciones conducentes a la adecuada } \\
\text { transferencia del conocimiento en diferentes escenarios como } \\
\text { empalmes, vacaciones, licencias, permisos, comisiones, inducciones, } \\
\text { entre otras. Periodicidad: trimestral. }\end{array}$ \\
\hline $\begin{array}{l}\text { Realizar trimestralmente una reunión general con todo el personal de } \\
\text { la Jefatura, que será dirigida por cada una de las direcciones de forma } \\
\text { rotativa, con el propósito de socializar experiencias asociadas a las }\end{array}$ \\
proyeriencias, \\
prón de
\end{tabular}

SIGNOS, Investigación en Sistemas de Gestión

ISSN: 2145-1389 | e-ISSN: 2463-1140 | DOI: https://doi.org/10.15332/24631140

Vol. 13 N.o 1 | enero-junio de 2021 


\begin{tabular}{ll}
\multicolumn{1}{c}{$\begin{array}{l}\text { Lograr que el conocimiento individual sea trasmitido y recibido entre los } \\
\text { servidores públicos para generar nuevos conjuntos de conocimientos y } \\
\text { experiencias. }\end{array}$} \\
$\begin{array}{ll}\text { conocimiento } \\
\text { entre direcciones }\end{array}$ & $\begin{array}{l}\text { diferentes direcciones para incrementar el conocimiento y las } \\
\text { competencias laborales. }\end{array}$ \\
\hline $\begin{array}{l}\text { Charlas de } \\
\text { aprendizaje entre } \\
\text { el personal de la } \\
\text { Jefatura }\end{array}$ & $\begin{array}{l}\text { Se requiere realizar charlas educativas entre el personal que } \\
\text { conforma la Jefatura e invitar al personal de otras Jefaturas que esté } \\
\text { interesado en aprender sobre diferentes áreas del conocimiento, core } \\
\text { del negocio, entre otras. Dichas charlas serán lideradas por los } \\
\text { funcionarios que tengan competencias, experiencia específica o } \\
\text { dominio de temas. } \\
\text { Se deberá hacer un listado con el personal que tenga dichas } \\
\text { competencias en temas de interés y hacer la programación respectiva } \\
\text { de manera semestral. }\end{array}$ \\
$\begin{array}{l}\text { Las competencias del personal que dictará las charlas deberán ser } \\
\text { validadas por la Dirección de Personal. }\end{array}$
\end{tabular}

Fuente: elaboración propia.

\section{Fase de exteriorización}

Esta dimensión tiene como propósito la interacción colectiva, es decir, tener conversaciones en equipo para formar conceptos (tabla 4).

Tabla 4. Fase de exteriorización

\begin{tabular}{|c|c|}
\hline \multicolumn{2}{|c|}{$\begin{array}{l}\text { Generar, crear, innovar y compartir nuevas ideas para el desarrollo de nuevos } \\
\text { conceptos y conocimiento. }\end{array}$} \\
\hline $\begin{array}{l}\text { Reunión de } \\
\text { funcionarios para } \\
\text { entrega de cargos } \\
\text { por dirección }\end{array}$ & $\begin{array}{l}\text { El gestor de transferencia de conocimiento de la dirección verificará } \\
\text { por medio de una reunión que la entrega de cargo entre los } \\
\text { funcionarios contenga los elementos claros para el desarrollo de las } \\
\text { funciones y el conocimiento del cargo y la Jefatura. El proceso debe } \\
\text { ser de forma adecuada en términos de tiempo, contenido y } \\
\text { pertinencia. } \\
\text { Presentar un documento que contenga la información detallada de } \\
\text { los resultados y las posibles acciones de mejora para el desarrollo de } \\
\text { dicho proceso. } \\
\text { Realizar acompañamiento y seguimiento al funcionario en las dos } \\
\text { semanas posteriores al ingreso, y una evaluación para reforzar los } \\
\text { conocimientos adquiridos durante y después de haber recibido la } \\
\text { capacitación o entrega del cargo. }\end{array}$ \\
\hline $\begin{array}{l}\text { Mesas de trabajo } \\
\text { con retos de } \\
\text { innovación para }\end{array}$ & $\begin{array}{l}\text { Desarrollar metodologías que promuevan la participación de los } \\
\text { colaboradores en el levantamiento de información y la mejora de los } \\
\text { diferentes documentos, procesos y retos institucionales. }\end{array}$ \\
\hline
\end{tabular}

SIGNOS, Investigación en Sistemas de Gestión

ISSN: 2145-1389 | e-ISSN: 2463-1140 | DOI: https://doi.org/10.15332/24631140

Vol. 13 N.o 1 | enero-junio de 2021 


\begin{tabular}{|c|c|}
\hline Generar, crear, inn & $\begin{array}{l}\text { Interacción colectiva de conocimiento } \\
\text { var y compartir nuevas ideas para el desarrollo de nuevos } \\
\text { conceptos y conocimiento. }\end{array}$ \\
\hline $\begin{array}{l}\text { transferencia de } \\
\text { conocimiento }\end{array}$ & $\begin{array}{l}\text { Realizar una mesa de trabajo bimestral entre el equipo de } \\
\text { funcionarios de la dirección para presentar propuestas de } \\
\text { mejoramiento del proceso. } \\
\text { Como resultado de la ejecución de la mesa de trabajo, quedará un } \\
\text { documento donde se especifiquen las acciones de mejoramiento, los } \\
\text { métodos de seguimiento y los responsables }\end{array}$ \\
\hline $\begin{array}{l}\text { Comités } \\
\text { interdisciplinarios } \\
\text { de transferencia de } \\
\text { conocimiento }\end{array}$ & $\begin{array}{l}\text { Realizar un comité semestral de transferencia de conocimiento con } \\
\text { grupos interdisciplinarios de las diferentes direcciones para } \\
\text { consolidar ideas que propendan por la mejora continua de los } \\
\text { procesos. } \\
\text { Se podrán tratar temas interrelacionados derivados de las mesas de } \\
\text { trabajo realizadas por cada dirección. } \\
\text { Del comité quedará como soporte un documento donde se describan } \\
\text { las tareas a realizar por cada dirección. } \\
\text { El director dará instrucciones al personal bajo su mando para la } \\
\text { ejecución de las actividades propuestas. } \\
\text { Realizar seguimiento a las acciones resultantes de los comités. }\end{array}$ \\
\hline $\begin{array}{l}\text { Reunión de } \\
\text { seguimiento y } \\
\text { análisis de } \\
\text { información }\end{array}$ & $\begin{array}{l}\text { Realizar reuniones de seguimiento para verificar la mejora de los } \\
\text { procesos por parte del personal a cargo con base en los indicadores. } \\
\text { Como soporte se deberán dejar actas de reunión. } \\
\text { Estas reuniones se harán con la periodicidad que se requieran, de } \\
\text { acuerdo a las necesidades. }\end{array}$ \\
\hline Fase de combir & ación \\
\hline $\begin{array}{l}\text { Esta dimensión tien } \\
\text { convertir el conocim }\end{array}$ & $\begin{array}{l}\text { como propósito la interacción colectiva, es decir, } \\
\text { ento en nuevas formas explícitas (tabla 5). }\end{array}$ \\
\hline Tabla 5. Fase de com & nación \\
\hline & Combinación del conocimiento \\
\hline Emplear herramient & $\begin{array}{l}\text { s tecnológicas para la transformación y desarrollo de nuevo } \\
\text { conocimiento accesible. }\end{array}$ \\
\hline $\begin{array}{l}\text { Espacio virtual de } \\
\text { transferencia de } \\
\text { conocimiento } \\
\text { institucional }\end{array}$ & $\begin{array}{l}\text { Promover el uso herramientas virtuales que permitan transferir el } \\
\text { conocimiento en todos los niveles de la entidad, por ejemplo: mensajes } \\
\text { institucionales con información relevante de conocimiento general para todo } \\
\text { el personal que conforma la Jefatura, campañas vía web con tips de } \\
\text { transferencia del conocimiento para todo el personal, cursos virtuales sobre } \\
\text { transferencia del conocimiento en las plataformas disponibles: Blackboard, } \\
\text { Avafp, Sibufp, Ovas, entre otras. }\end{array}$ \\
\hline
\end{tabular}

SIGNOS, Investigación en Sistemas de Gestión

ISSN: 2145-1389 | e-ISSN: 2463-1140 | DOI: https://doi.org/10.15332/24631140

Vol. 13 N.o 1 | enero-junio de 2021 


\begin{tabular}{ll}
\hline \multicolumn{1}{|c|}{$\begin{array}{c}\text { Combinación del conocimiento } \\
\text { Emplear herramientas tecnológicas para la transformación y desarrollo de nuevo } \\
\text { conocimiento accesible. }\end{array}$} \\
$\begin{array}{l}\text { Directriz para } \\
\text { transferir el } \\
\text { conocimiento } \\
\text { institucional }\end{array}$ & $\begin{array}{l}\text { Fijar una directriz para transferir el conocimiento entre los funcionarios de la } \\
\text { institución, donde se estipulen sitios, horarios, frecuencia, cantidad } \\
\text { participantes, sesiones, tipos de actividades y otros factores que se consideren } \\
\text { oportunos. }\end{array}$ \\
$\begin{array}{l}\text { Esta directriz deberá ser formalizada a través de un documento oficial como } \\
\text { una directiva o circular, deberá ser divulgada por medio de los espacios } \\
\text { virtuales de conocimiento y el gestor deberá velar por su pleno cumplimiento. }\end{array}$ \\
$\begin{array}{l}\text { Se recomienda que el documento sea planteado por el área de planeación en } \\
\text { coordinación con talento humano. }\end{array}$ \\
\hline $\begin{array}{l}\text { Plataforma } \\
\text { documental } \\
\text { electrónica de } \\
\text { transferencia del } \\
\text { conocimiento }\end{array}$ & $\begin{array}{l}\text { Optimizar y fortalecer los medios de divulgación y socialización de la } \\
\text { información documentada a través de sistemas de información como Orfeo, } \\
\text { correo institucional y otros. }\end{array}$ \\
\hline $\begin{array}{l}\text { Stocks de } \\
\text { documentos por } \\
\text { dirección }\end{array}$ & $\begin{array}{l}\text { Diseñar documentos que fomenten la transferencia de conocimiento entre los } \\
\text { procesos de la Jefatura (procedimientos, instructivos, guías, demos, entre } \\
\text { otros). }\end{array}$ \\
\hline
\end{tabular}

Fuente: elaboración propia.

\section{Fase de interiorización}

Esta dimensión tiene como propósito apropiarse de nuevos conocimientos explícitos de la organización, es decir, interiorizar nuevo conocimiento explícito (tabla 6).

Tabla 6. Fase de interiorización

\begin{tabular}{|l|l|}
\multicolumn{1}{c}{$\begin{array}{l}\text { Interiorización del nuevo conocimiento } \\
\text { Retener e incorporar el nuevo conocimiento adquirido para su desarrollo y } \\
\text { aplicabilidad en las actividades diarias. }\end{array}$} \\
$\begin{array}{l}\text { Talleres prácticos } \\
\text { de transferencia } \\
\text { del conocimiento }\end{array}$ & $\begin{array}{l}\text { Incluir en el seminario de educación un taller práctico de transferencia del } \\
\text { conocimiento a todos los niveles de la organización, sin importar las } \\
\text { diferencias de cargos o jerarquías, donde se establezcan temas específicos, } \\
\text { actividades y casos especiales por procesos. } \\
\text { Realizar un plan de actividades del seminario teniendo en cuenta temas } \\
\text { relevantes, dificultades y retos que se tengan en los diferentes niveles de la } \\
\text { Jefatura. } \\
\text { Realizar una evaluación de la efectividad del seminario. }\end{array}$ \\
\hline
\end{tabular}

SIGNOS, Investigación en Sistemas de Gestión

ISSN: 2145-1389 | e-ISSN: 2463-1140 | DOI: https://doi.org/10.15332/24631140

Vol. 13 N.o 1 | enero-junio de 2021 


\begin{tabular}{ll}
\multicolumn{1}{c}{ Retener e incorporar el nuevo conocimiento adquirido para su desarrollo y } \\
aplicabilidad en las actividades diarias.
\end{tabular}

Fuente: elaboración propia.

Finalizada la propuesta, se validó el contenido con el propósito de establecer si las características de esta respondían a la necesidad planteada mediante la técnica de juicio de expertos. Para ello, se definieron como criterios para la selección de los expertos (tabla 7). La validación del contenido también se comprende como el grado en que un instrumento mide lo que se quiere medir o si cumple con el objetivo propuesto para el cual fue construido (Urrutia et ál., 2014).

Tabla 7. Definición de criterios para el perfil de validador de contenido

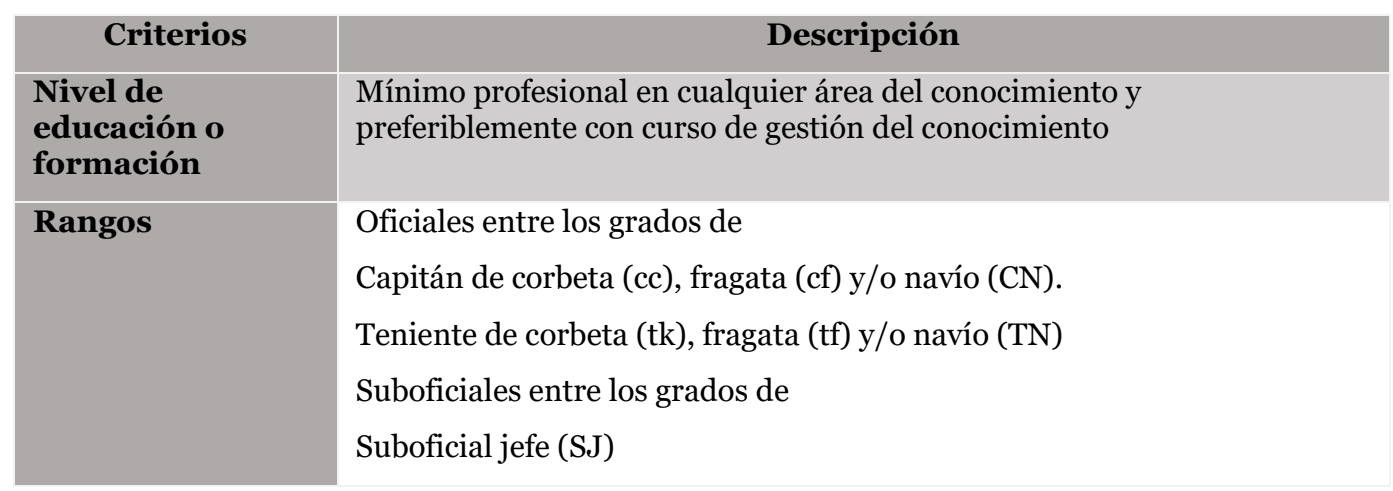




\begin{tabular}{|l|l|}
\hline \multicolumn{1}{|c|}{ Criterios } & \multicolumn{1}{c|}{ Descripción } \\
\hline Sargento viceprimero (SV) y sargento segundo (SS) \\
\hline Experiencia & $\begin{array}{l}\text { Mínimo un año comodirector de dependencia o jefe de sección y/o } \\
\text { división de cualquier área y unidad } \\
\text { Antigüedad }\end{array}$ \\
\hline De cinco años en adelante en la entidad \\
\hline
\end{tabular}

Fuente: elaboración propia.

El análisis fue efectuado por siete expertos que cumplían con los criterios y que aplicaron el instrumento de validación bajo las categorías de claridad, pertinencia y aplicabilidad. Los resultados generales se presentan en la tabla 8. Los cuestionarios se aplicaron en forma presencial y se establecieron los siguientes criterios para el análisis de los resultados:

- Se considera aceptable un ítem si las dos terceras partes del total de respuestas o más (66 \% o más) se encuentran en los niveles 5 (totalmente de acuerdo) o 4 (de acuerdo).

- Se considera que el enunciado es negativo si las dos terceras partes del total de respuestas o más (66 \% o más) se encuentran en los niveles 1 (totalmente en desacuerdo), 2 (en desacuerdo) y 3 (se requiere hacer ajustes).

Tabla 8. Resultados generales

\begin{tabular}{|c|c|c|c|c|c|c|c|c|c|c|}
\hline \multirow[t]{2}{*}{ Categorías } & \multirow[t]{2}{*}{ Ítems } & \multicolumn{3}{|c|}{$\begin{array}{c}\text { Criterios de } \\
\text { respuesta no } \\
\text { aceptable }\end{array}$} & \multirow[t]{2}{*}{ Total } & \multicolumn{2}{|c|}{$\begin{array}{c}\text { Criterios de } \\
\text { respuesta } \\
\text { aceptable }\end{array}$} & \multirow[t]{2}{*}{ Total } & \multicolumn{2}{|c|}{$\begin{array}{l}\text { Resultados } \\
\text { de } \\
\text { aceptación }\end{array}$} \\
\hline & & 1 & 2 & 3 & & 4 & 5 & & Sí & No \\
\hline \multirow[t]{3}{*}{ Claridad } & 1 & & & & O & $29 \%$ & $71 \%$ & $100 \%$ & $x$ & \\
\hline & 2 & & & & o & & $100 \%$ & $100 \%$ & $x$ & \\
\hline & 3 & & & & o & & $100 \%$ & $100 \%$ & $x$ & \\
\hline \multirow[t]{2}{*}{ Pertinencia } & 4 & & & & o & $14 \%$ & $86 \%$ & $100 \%$ & $x$ & \\
\hline & 5 & & & & 0 & $43 \%$ & $57 \%$ & $100 \%$ & $x$ & \\
\hline
\end{tabular}




\begin{tabular}{|c|c|c|c|c|c|c|c|c|c|c|}
\hline \multirow[t]{2}{*}{ Categorías } & \multirow[t]{2}{*}{ Ítems } & \multicolumn{3}{|c|}{$\begin{array}{l}\text { Criterios de } \\
\text { respuesta no } \\
\text { aceptable }\end{array}$} & \multirow[t]{2}{*}{ Total } & \multicolumn{2}{|c|}{$\begin{array}{c}\text { Criterios de } \\
\text { respuesta } \\
\text { aceptable }\end{array}$} & \multirow[t]{2}{*}{ Total } & \multicolumn{2}{|c|}{$\begin{array}{l}\text { Resultados } \\
\text { de } \\
\text { aceptación }\end{array}$} \\
\hline & & 1 & 2 & 3 & & 4 & 5 & & Sí & No \\
\hline & 6 & & & & $\mathrm{O}$ & $29 \%$ & $71 \%$ & $100 \%$ & $x$ & \\
\hline \multirow[t]{4}{*}{ Aplicabilidad } & 7 & & & & O & $57 \%$ & $43 \%$ & $100 \%$ & $x$ & \\
\hline & 8 & & & & O & $29 \%$ & $71 \%$ & $100 \%$ & $x$ & \\
\hline & 9 & & & & 0 & $29 \%$ & $71 \%$ & $100 \%$ & $x$ & \\
\hline & 10 & & & & 0 & $43 \%$ & $57 \%$ & $100 \%$ & $x$ & \\
\hline
\end{tabular}

Fuente elaboración propia.

Se encontró que de acuerdo con el juicio de los expertos, todos los ítems con aceptables en cuanto están por encima del $66 \%$, lo cual indica que la estrategia planteada es clara, pertinente y aplicable para las necesidades de la Jefatura. Las observaciones que realizaron algunos de los expertos se incorporaron al documento, entre las cuales se encuentran:

- $\quad$ Ajustar el párrafo de presentación de la estrategia.

- Buscar un sinónimo de la palabra tácito para que sea comprensible.

- Ser más específicas las actividades para la Jefatura y las direcciones.

- Incluir dentro de los espacios virtuales los nombres de las plataformas existentes como complemento.

- Incluir una evaluación en las actividades propuestas en reunión de funcionarios para entrega de cargos por dirección.

- Cambiar la palabra $B a$ por espacios de conocimiento para que sea comprensible.

- Tener en cuenta el plan de capacitación anual para formación en transferencia del conocimiento.

Con el fin de evaluar la fiabilidad del juicio de expertos, se calcularon el coeficiente de correlación alfa de Cronbach y el coeficiente de concordancia W de Kendall, a partir de los datos que consignaron los siete expertos. El coeficiente alfa de Cronbach permite estimar la confiabilidad 
de un instrumento a través del análisis del perfil de las respuestas dadas y la correlación media entre las preguntas. Es decir, es el promedio de la suma de todos los coeficientes de las respuestas dadas por cada experto. Los resultados de este coeficiente pueden variar en una escala de o y 1 , siendo un resultado de coeficiente alfa $>0.9$ excelente $y$, por el contrario, un resultado <0.5 inaceptable (George y Mallery, 1995). El análisis se efectuó mediante una aplicación en tablas dinámicas de Excel y arrojó un valor de 0.795, que es considerado aceptable, por lo cual se considera que desde esta óptica la propuesta es confiable.

El coeficiente de concordancia W de Kendall se usa para determinar el grado de asociación entre tres o más variables del constructo de una prueba. El instrumento para evaluar la validez de contenido tiene tres categorías: claridad (tres ítems), pertinencia (tres ítems) y aplicabilidad (cuatro ítems). El objetivo de este estadístico es capturar la varianza y no el número o valor de la calificación. El análisis se efectuó mediante una aplicación en tablas dinámicas de Excel y arrojó los resultados que muestra la tabla 9 .

Tabla 9. Coeficiente de concordancia W de Kendall

\begin{tabular}{|l|l|l|l|l|}
\hline $\begin{array}{c}\text { Datos/criter } \\
\text { ios }\end{array}$ & \multicolumn{1}{|c|}{ Convenciones } & \multicolumn{1}{|c|}{ Claridad } & $\begin{array}{l}\text { Pertinenc } \\
\text { ia }\end{array}$ & $\begin{array}{c}\text { Aplicabilid } \\
\text { ad }\end{array}$ \\
\hline $\mathbf{N}$ & Número de ítems (columna) & 3 & 3 & 4 \\
\hline $\mathbf{M}$ & Número de expertos (fila) & 7 & 7 & 7 \\
\hline $\mathbf{W}$ & $\begin{array}{l}\text { Coeficiente de concordancia W de } \\
\text { Kendall }\end{array}$ & $\begin{array}{l}0.1269841 \\
27\end{array}$ & $\begin{array}{l}0.0612244 \\
9\end{array}$ & 0.035714286 \\
\hline$\chi^{2}$ calculada & Distribución de chi-cuadrado & 1.77777777 & 0.8571428 & 0.75 \\
\hline$\chi^{2}$ Tabla & & 8 & 57 & 13.85 \\
\hline
\end{tabular}

Fuente: elaboración propia.

El coeficiente W de Kendall se basa en la distribución no paramétrica chicuadrado y permite establecer dos hipótesis: 


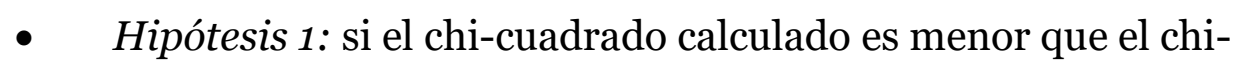
cuadrado de la tabla, los siete expertos están de acuerdo en su opinión sobre los ítems del criterio claridad o de la categoría que se está evaluando.

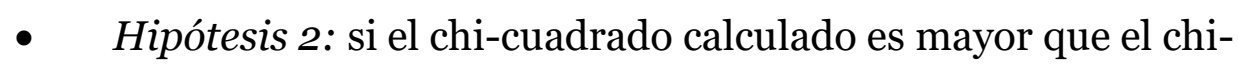
cuadrado de la tabla, los siete expertos no están de acuerdo en su opinión sobre los ítems del criterio claridad o de la categoría que se está evaluando.

Se observa la siguiente concordancia entre las categorías:

- Claridad: el valor de chi-cuadrado calculado, según los datos arrojados por la herramienta estadística a partir de la evaluación de los siete expertos, es de 1.77. Al comparar este valor con el chicuadrado de la tabla, que es de 9.39, se acepta la hipótesis 1: los expertos están de acuerdo en su opinión sobre los tres ítems evaluados para esta categoría.

- Pertinencia: el valor del chi-cuadrado calculado, de acuerdo con los datos que calcula la herramienta estadística a partir de la evaluación de los siete expertos, es de 0.85 . Al comparar este valor con el chicuadrado de la tabla, que es de 13.85, se acepta la hipótesis 1: los expertos están de acuerdo en su opinión sobre los tres ítems evaluados para esta categoría.

- $\quad$ Aplicabilidad: el valor del chi-cuadrado calculado, según los datos que calcula la herramienta estadística a partir de la evaluación de los siete expertos, es de 0.75, al comparar este valor con el chi-cuadrado de la tabla, que es de 13.8, se acepta la hipótesis 1: los expertos están de acuerdo en su opinión sobre los tres ítems evaluados para esta categoría. 
Por lo anterior, se determina que para las tres categorías se acepta la hipótesis 1; es decir, existe concordancia en la evaluación de los expertos y, por lo tanto, los resultados de la evaluación de las categorías claridad, pertinencia y aplicabilidad son confiables.

La investigación demostró que este es un campo emergente en el cual no hay todavía un marco consensuado y sólido (Dayan y Evans, 2006, Brix, 2017). Se encontraron múltiples referencias sobre las ventajas que tiene para las organizaciones implantar estrategias efectivas para gestionar el conocimiento, considerándolo un factor para el éxito tan importante como los recursos financieros, físicos o humanos (Hong et ál., 2011, Serenko y Bontis, 2016).

Sin embargo, Viedema (2006) afirma que no todos los conocimientos generan ventaja competitiva, sino solo aquellos que contribuyen a la generación de valor económico y que constituyen un conocimiento pertinente. Tissen et ál. (2000) señalan que el conocimiento agrega valor por la eficiencia del conocimiento - la facilidad que tienen las personas para acceder a un conocimiento pertinente y actualizado-, la conectividad del conocimiento - la capacidad organizacional para que se desarrolle conocimiento colaborativo- y la innovación en el conocimiento - la capacidad para combinar en formas novedosas el conocimiento-. Un aspecto determinante en la gestión del conocimiento es la importancia que se da entre conocimiento tácito y conocimiento explícito; el conocimiento tácito radica en las personas, es contextual y, por lo tanto, difícil de comunicar y de formalizar, mientras que el conocimiento explícito es un conocimiento codificado, que puede ser transmitido por algún tipo de lenguaje (Nonaka y Takeuchi, 1995).

El conocimiento tácito se encuentra dentro de las habilidades de las personas, en sus mapas mentales, surge con la experiencia y requiere un trabajo adicional para exteriorizarlo y plasmarlo en palabras, algoritmos o 
formas físicas. Un aspecto novedoso en la teoría de la creación del conocimiento que plantean Nonaka y Takeuchi (1995), y que se decidió utilizar como referente en este estudio, es que la creación de conocimiento se ha convertido en un modelo dinámico que se contextualiza en un entorno social, es decir, necesita de la interacción entre las personas, no unidireccional, sino en forma de una espiral (Acosta et ál., 2014). Como punto de partida para implementar una estrategia para la gestión del conocimiento en las organizaciones se requiere identificar, conocer y valorar los recursos disponibles en la organización (Barney, 1991), así como las prácticas que habitualmente se desarrollan en relación con el conocimiento y su transformación de tácito en explícito.

La transferencia del conocimiento, de acuerdo con los modelos que la entidad escoja implementar, también contribuyen a aumentar la innovación de sus trabajadores, debido a que los conocimientos que son compartidos hacia otras personas servirán como referentes para mejorarlos continuamente (Zabala y Quintero, 2017). También se entiende en el contexto de este estudio como la capacidad de la organización para asegurar que aquel conocimiento tácito relevante para desarrollar las funciones clave de un cargo, se le entregue al sucesor del cargo en el momento en que se presente un cambio, de manera eficiente y eficaz.

\section{Conclusiones}

El problema identificado como punto de partida del estudio fue la pérdida de conocimiento en la operación y el control de los procesos y las actividades administrativas de la Jefatura, debido a la alta rotación anual de personal militar y civil en los cargos. Esta situación se presenta de manera rutinaria por traslados, ascensos y retiros, y forma parte de la cultura organizacional del sector defensa. Se encontró que la rotación anual de personal entre oficiales, suboficiales, infantes de marina y 
personal civil en los cinco últimos años variaba entre 40-50 \% y la ausencia de estrategias efectivas para asegurar la transferencia de conocimiento en estas situaciones generaba tensiones administrativas, incumplimientos y recargas de trabajo.

Se realizó una revisión de literatura que permitió identificar las cinco principales teorías que han surgido en los últimos 30 años sobre la gestión del conocimiento y sus particularidades en relación con la transferencia del conocimiento. De esta revisión, se seleccionó la teoría de la creación del conocimiento propuesta por Nonaka y Takeuchi (1995), por su enfoque en la organización como sistema y en la secuencia cíclica para el mejoramiento continuo, los cuales son compatibles con los sistemas de gestión basados en normas técnicas internacionales. Esta teoría brindó las bases para efectuar el diagnóstico en la Jefatura de Formación, Instrucción y Educación Naval de la Armada Nacional (Jinen) y sustentó la propuesta de mejora para la transferencia de conocimiento en la entidad.

Con el propósito de evaluar el estado actual de la entidad, se aplicó el instrumento de diagnóstico de la gestión del conocimiento (Peña, 2015). A partir de las cuatro fases del modelo SECI (socialización, exteriorización, combinación e interiorización), planteadas en la teoría de la creación del conocimiento organizacional, este instrumento permitió determinar que la Jefatura tiene dificultades para gestionar su conocimiento en las fases de exteriorización, combinación e interiorización, mas no en la de socialización. Además, existen algunos Ba establecidos como espacios para gestionar conocimiento, pero la organización no los identifica como tales, y existen debilidades en los facilitadores organizacionales, excepto en el de intención. Del análisis se concluye que el facilitador de autonomía de las personas no es aplicable en el contexto de la Armada Nacional y los facilitadores de fluctuación y caos creativo, redundancia y variedad de requisitos no se verán reflejados directamente con estos nombres dentro 
de la propuesta, sino que se incluirán con otros nombres para hacerlos comprensibles para la organización y darle dinamismo a la estrategia.

A partir de los resultados del diagnóstico y con base en los fundamentos teóricos analizados, se planteó una estrategia para la transferencia del conocimiento en la Jefatura que le ayude a solucionar la necesidad actual. Esta propuesta le permitirá a la entidad transferir adecuadamente el conocimiento entre funcionarios a través de unas acciones aplicables, pertinentes, claras y comprensibles, tales como nombramiento de un gestor de transferencia del conocimiento para un periodo determinado por cada una de las cinco direcciones que tiene la Jinen, campañas de divulgación, reunión de entrega de cargos entre funcionarios y el gestor, entre otras. Estas acciones se organizan en cuatro componentes que articulan las fases de la gestión del conocimiento en un lenguaje más cercano a los usuarios de la Jefatura: difusión de conocimiento individual, interacción colectiva de conocimiento, combinación del conocimiento e interiorización del nuevo conocimiento. El propósito de la propuesta es modificar aspectos de la cultura organizacional que impiden una adecuada transferencia del conocimiento.

La propuesta se sometió a un proceso de validación de contenido a partir de una metodología planteada por Rodríguez-Rojas (2017) para recoger las percepciones de siete expertos frente a los planteamientos presentados en la estrategia. Estos expertos cumplían los requisitos previamente establecidos de experiencia, antigüedad en la Armada, educación y conocimientos. Los resultados que brindó la evaluación por los expertos se sometieron a pruebas para verificar su confiabilidad estadística, por medio de la aplicación del coeficiente de correlación alfa de Cronbach, el cual arrojó un resultado del o.8 de confiabilidad, que según la literatura es aceptable, y del coeficiente de concordancia W de Kendall, que concluyó 
que las categorías de claridad, pertinencia y aplicabilidad planteadas para la propuesta son fiables.

Como resultado de la validación de contenido de la estrategia, surgieron algunas observaciones y una sugerencia por parte de los expertos que mejoraron el planteamiento de la estrategia, para responder a las necesidades de la Jefatura. Por medio del planteamiento de la estrategia se espera impactar, en primera instancia, a la Jinen, conformada actualmente por 59 funcionarios. A mediano plazo, el impacto podría ser mayor si esta estrategia se proyecta a las demás jefaturas de la Armada Nacional, alcanzando a los 34600 funcionarios con los que cuenta actualmente la entidad. A largo plazo, podría lograr un impacto global en el sostenimiento y mejoramiento continuo de todos los procesos de la institución.

\section{Referencias}

Acosta, J., Zárate, R. y Fischer, A. (2014). Ba: espacios de conocimiento. contexto para el desarrollo de capacidad de innovación. Un análisis desde la gestión del conocimiento. Revista Escuela de Administración de Negocios, 76, 44-63.

https://doi.org/10.21158/01208160.n76.2014.797

Barney, J. B. (1991). Firm resources and sustained competitive advantage. Journal of Management, 17, 99-120.

Brix, J. (2017). Exploring knowledge creation processes as a source of organizational learning: a longitudinal case study of a public innovation project. Scandinavian Journal of Management, 33(2), 113-127.

Bueno, E. (2003). El reto de emprender en la sociedad del conocimiento: el capital de emprendimiento como dinamizador del capital intelectual. En E. Genescá et ál. (coords.), Creación de empresas - Entrepreneurship. Universitat Autònoma de Barcelona.

Dayan, R. y Evans, S. (2006). KM your way to CMMI. Journal of Knowledge Management, 10(1), 69-80. 
Engelbrecht, A., Gerlach, J., Benlian, A. y Peter, B. (2019). How employees gain metaknowledge using enterprise social networks: a validation and extension of communication visibility theory. Journal of Strategic Information Systems, 28(3), 292-309. https://doi.org/10.1016/j.jsis.2019.04.001

George, D. y Mallery, P. (1995). SPSS/PC+ step by step: a simple guide and reference. Wadsworth.

Hong, D., Suh, E. y Koo, C. (2011). Developing strategies for overcoming barriers to knowledge sharing based on conversational knowledge management: a case study of a financial company. Expert Systems with Applications, 38(12), 14417-14427. https://doi.org/10.1016/j.eswa.2011.04.072.

Maier, R. (2007). Knowledge management systems. Springer.

Marulanda, C., Bedoya, O. y Quintero, H. (2018). Modelo de transferencia del conocimiento para centros e institutos de investigación. Revista Espacios, 39(17), 35 .

Nonaka, I. y Takeuchi, H. (1995). La organización creadora de conocimiento. Oxford University Press.

Organización Internacional para la Estandarización. (2015). ISO 9001:2015.

Peña, G. (2015). Diagnóstico de la gestión del conocimiento. Notas de la asignatura Gestión Integral de la Maestría en Calidad y Gestión Integral. Universidad Santo Tomás.

Rodríguez, Y. (2017). Evaluación de la madurez de la gestión de la seguridad y salud en el trabajo en universidades con acreditación de alta calidad multicampus de Bogotá [Tesis de doctorado]. Universidad de Celaya.

Romero, V. (2018, 30 de agosto). ¿Por qué es importante la gestión del conocimiento en las empresas? Ruiz Healy Times. https://ruizhealytimes.com/economia-ynegocios/por-que-es-importante-la-gestion-del-conocimiento-en-las-empresas/

Santamaría, G. y Cárdenas, M. (2016). La transferencia del conocimiento en las empresas. UTCiencia, 3(1), 21-34.

Serenko, A. y Bontis, N. (2016). Understanding counterproductive knowledge behavior: Antecedents and consequences of intra-organizational knowledge hiding. Journal of Knowledge Management, 2O(6), 1199-1224.

SIGNOS, Investigación en Sistemas de Gestión

ISSN: 2145-1389 | e-ISSN: 2463-1140 | DOI: https://doi.org/10.15332/24631140

Vol. 13 N. ${ }^{\circ} 1$ | enero-junio de 2021 
Tissen, R., Andriessen, D. y Lekanne, F. (2000). El valor del conocimiento para aumentar el rendimiento en las empresas. Prentice Hall.

Torres, C. L. y Rojas, R. S. (2017). La gestión del conocimiento basado en la teoría de Nonaka y Takeuchi. INNOVA Research Journal, 2(4), 30-37. https://doi.org/10.3389o/innova.v2.n4.2017.147

Urrutia, M., Barrios, S., Gutiérrez, M. y Mayorga, M. (2014). Métodos óptimos para determinar validez de contenido. Educación Médica Superior, 28(3), 547-558.

Vázquez, E. (2017). Transferencia del conocimiento y tecnología en universidades. Iztapalapa, 38(83), 75-95.

Viedma, J. (2001). ICBS Intellectual Capital Benchmarking Systems. Journal of Intellectual Capital, 2(2), 148-164.

Zabala, D. y Quintero, S. (2017). Modelos de gestión para la transferencia de los conocimientos en instituciones de educación superior. Revista Ciencias Estratégicas, 25(38), 441-456. 